\title{
Análisis
}

\section{Desarrollo económico y medio ambiente: el caso de Malasia}

DOI: 10.32870/mycp.v10i30.292

Ana Bertha Cuevas Tello*

\section{Introducción}

$\mathrm{E}$ $n$ los últimos años ha surgido una discusión, no acotada, sobre la estrecha relación que existe entre el desarrollo económico y el medio ambiente. En este mismo recorrer del tiempo, tanto el crecimiento económico global como el deterioro ambiental han presentado tasas de aumento sin precedentes. El producto interno bruto (PIB) mundial pasó de 14.3 miles de millones de dólares en 1970 a 40.2 en $2004 .{ }^{1}$ Es decir, durante estos 34 años la economía mundial presentó un crecimiento promedio anual de $3 \%$. Mientras que el incremento de gases de efecto invernadero (generadores del cambio climático), las tasas de deforestación de los bosques, la destrucción de la capa de ozono, la extinción de algunas especies de flora y fauna son algunos de los ejemplos que explican la degradación paulatina del medio ambiente global.

Algunos especialistas aluden a que el crecimiento económico tiene un efecto

* Profesora investigadora del Departamento de Estudios del Pacífico de la Universidad de Guadalajara. ORCID http:// orcid.org/0000-0002-4553-8141 negativo sobre la calidad del medio ambiente. ${ }^{2}$ Otros afirman que los habitantes de los países desarrollados tienden a valorar más la naturaleza en la medida que su ingreso es más alto. ${ }^{3}$ Mientras que otro grupo defiende la tesis de que es el sistema económico actual (capitalista) el único responsable de la severa degradación del entorno natural presente. ${ }^{4}$

Los economistas afirman que el crecimiento económico permite proporcionar a los ciudadanos mejores niveles de consumo, acceso a servicios de salud, la educación, elevar la esperanza de vida, incrementar las pensiones públicas para los jubilados, entre otros beneficios. ${ }^{5}$ En síntesis, la postura economicista señala al crecimiento económico como la única vía para mejorar la calidad de vida de los habitantes del mundo.

Sin embargo, estudios recientes sobre el medio ambiente señalan que los problemas globales como el cambio climático podrían afectar los elementos básicos de la vida de la gente tales como el acceso al agua, la producción de alimentos, la salud y el ambiente. Agregan que cientos de millones de personas podrían sufrir hambre, escasez de agua o inundaciones y — subrayan — que

MÉXICO YLACUENCADEL PACÍFICO vol. 10, núm. 30 / septiembre-diciembre de 2007 
si no se toman las medidas inmediatas correspondientes los costos económicos podrían ser muy altos. ${ }^{6}$ Es decir, el descuido del medio ambiente amenaza no sólo con disminuir la calidad de vida o, incluso, terminar con ella, sino que pone en riesgo el tan apreciado crecimiento económico que sustenta la sociedad actual.

La discusión anterior permite intuir la cercana relación entre las dos variables y su complejidad; la preponderancia de una (crecimiento económico) como causante de la otra (deterioro ambiental) y viceversa; la amenaza que vive la sociedad como medio de acción para la continuidad del sistema y como receptora de los efectos del mismo.

Bajo este singular contexto, Malasia se presenta como un caso interesante de analizar pues este país se distingue, a partir de la segunda mitad de los años setenta, por ser una de las naciones con mayor desarrollo económico en el mundo, por la riqueza de sus recursos naturales y por los problemas de contaminación de los que se ha visto acompañado en el camino a su industrialización. ${ }^{7}$

Con base en lo anterior resulta interesante analizar esta serie de interrogantes ¿De dónde surge la relación crecimiento económico con el medio ambiente? ¿Es el deterioro ambiental consecuencia del crecimiento económico? ¿Perjudica la protección del medio ambiente a la economía? ¿Por qué se dice que el sistema capitalista es el causante del deterioro ambiental? ¿Es el desarrollo sustentable una opción viable? ¿Es el caso de Malasia una representación micro del acontecimiento global?

Éstas son algunas de las interrogantes que se intentará resolver a lo largo de este trabajo. En el primer apartado se analizarán las características económicas de los recursos naturales y su papel como insumo económico. En el segundo se realizará un debate teórico sobre la relación crecimiento económico y medio ambiente, a la vez que se analizará la propuesta del desarrollo sustentable como una vía alternativa. Por último, se realizará un análisis del caso de Malasia que permita encontrar relación o no entre el crecimiento económico y el deterioro ambiental.

\section{Características económicas de los recursos naturales y el medio ambiente como insumo económico}

Los recursos naturales y el medio ambiente son factores de producción: a) la tierra proporciona alimento, minerales, combustibles fósiles, bosques, etc.; b) el agua es la fuente de vida, suministra una diversidad de víveres, actividades recreativas, funge como medio de transporte, entre otras; c) la atmósfera brinda aire respirable, puestas de sol y espacio para traslado de aviones. ${ }^{8}$

Bajo esta perspectiva, donde se combinan elementos tanto de sobrevivencia como de producción y servicio, los economistas clasifican los recursos naturales en cuatro categorías: a) apropiables o inapropiables; b) renovables y no renovables; c) esenciales o no esenciales, y d) privados o públicos y público mundiales.

Se dice que un recurso natural es apropiable cuando el dueño del bien o el consumidor pueden recoger el valor económico del producto, es decir, se obtiene un beneficio monetario de ello o una satisfacción interior; ejemplo de esto son los combustibles energéticos, las piedras preciosas, los árboles, etc. Mientras que los bienes inapropiables generan costos y beneficios que no van a parar solamente a sus propietarios o a los consumidores, pues generan externalidades que no se reflejan "totalmente en los precios y en las transacciones del mercado"; 9 prueba de éstos son los bosques, ya que los 
árboles no sólo tienen un valor económico sino que dentro de sus funciones están las de generar oxigeno, regular el balance de agua y la temperatura de la tierra (al facilitar los mantos freáticos), además de fungir como hogar de una gran diversidad biológica y como fuente de recreación. ${ }^{10} \mathrm{En}$ otras palabras, cuando se corta un árbol para la producción, el mercado no se apropia o recoge todos los beneficios de este bien, ni se responsabiliza de los males que ocasiona; en este sentido, se generan externalidades negativas en el mundo de las que nadie se hace responsable.

Los recursos naturales son renovables cuando sus servicios se reponen periódicamente o tienen capacidad de renovación propia (suelo agrícola, madera, energía solar). ${ }^{11}$ Un recurso no renovable ${ }^{12}$ "es aquel cuya oferta es esencialmente fija o no se regenera suficientemente de prisa"; ${ }^{13}$ entre éstos tenemos el oro, la plata, el petróleo, el gas, entre otros.

Los recursos naturales que no tienen sustitución, como el oxigeno, el agua, los bosques (por la absorción de dióxido de carbono, aire limpio) son considerados bienes esenciales, puesto que no hay otro elemento que los supla. En cambio, a todos los recursos que tienen reemplazo se les considera como no esenciales. Ejemplo, los combustibles fósiles como el petróleo y el gas, pues se pueden sustituir por carbón, y cuando este último se agote se sustituye con energía nuclear o solar.

Los bienes naturales públicos mantienen dos cualidades fundamentales: "sus beneficios no presentan rivalidad en su consumo y no son excluibles"; ${ }^{14}$ además sus beneficios se difunden indivisiblemente por toda la comunidad, independientemente de que las personas deseen o no comprarlos; ${ }^{15}$ muestra de esto son las externalidades positivas de los bosques. Por otro lado, los bienes privados tienen la característica de "que se pueden dividir y suministrarse por separado a diferentes individuos sin que produzcan beneficios ni costes externos a otros". ${ }^{16}$ Por último, dentro de los recursos naturales existen bienes públicos mundiales, los cuales son aquellos cuyas externalidades se difunden indivisiblemente por todo el planeta.

Hasta aquí se ha presentado la importancia de los recursos naturales en la economía y la clasificación de éstos. Es clara la dependencia que tiene el sistema económico de los recursos naturales tanto como bienes de producción como de servicio. Evidentemente el primero se sostiene del segundo, pues la naturaleza provee la materia prima y la energía que hace funcionar al sistema. Sin embargo, existe otra relación que va más allá del uso que el sistema de producción hace de los recursos naturales, es decir, lo que el modelo económico le regresa al medio ambiente.

Conforme a Field y Field, "las funciones económicas básicas que se realizan en una sociedad son la producción y el consumo". Estas actividades generan durante su marcha productos de desecho (residuos) que invariablemente regresan, en el corto o largo plazo, a la naturaleza, hecho que genera contaminación o degradación al medio ambiente. ${ }^{17}$

La gráfica 1 ilustra la interrelación de la naturaleza con la economía. ${ }^{18}$ Se puede apreciar cómo la naturaleza es el soporte de la economía y cómo esta última vacía, durante el proceso de producción, sus residuos al medio ambiente. En este sentido, la producción y el consumo generan residuos que van a parar de manera directa al aire, al agua o la tierra.

El modelo anterior refleja el funcionamiento simple del sistema economía-naturaleza. En la actualidad existe una diversi-

MÉXICO YLACUENCADEL PACÍFICO vol. 10, núm. 30 / septiembre - diciembre de 2007 


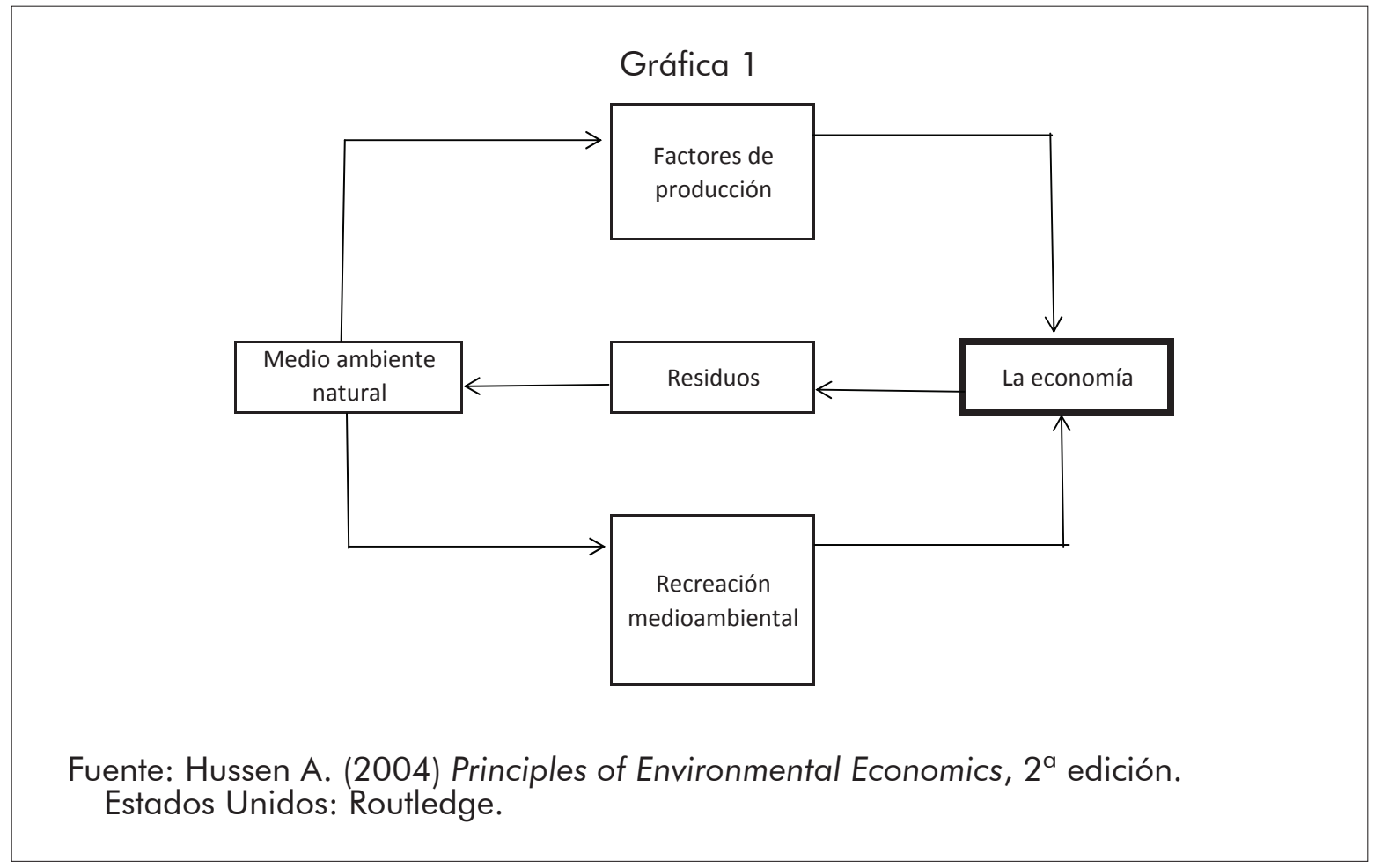

dad de propuestas de gestión de los flujos de residuos que intentan reducir el daño ambiental y que al mismo tiempo agregan complejidad al modelo. No obstante, la gráfica es pertinente pues permite entender el proceso. Si posteriormente se le agregan las medidas de reciclaje, tecnología limpia o reducción de producción y consumo, se verá que los residuos disminuyen pero no desaparecen del todo, pues como Uranga lo menciona, "el reciclaje industrial implica la dilapidación de más recursos para hacer útil lo ya degradado, y en su andar genera otros residuos que suelen pasarse por alto en el conteo final".

Bajo este análisis, es relevante señalar que la tierra tiene una capacidad finita de asimilación de contaminantes, por lo que no es inmune a los residuos; en otras palabras, la naturaleza está dotada con capacidad para acoger ciertos elementos contaminantes y convertirlos en benignos o inofensivos, empero esta características no es infinita por lo que poco a poco se está minando la calidad del entorno. ${ }^{19}$

Hasta aquí se ha presentado el manejo que la economía hace del medio ambiente. Es evidente, con base en los elementos analizados, que conforme aumenten los niveles de producción y consumo aumentará la degradación del ambiente, y que además los recursos naturales con los que cuenta el planeta no serán permanentes (incluso los renovables). ${ }^{20}$ Bajo estas condiciones esto representaría, en un futuro no muy lejano, un problema de proveeduría y una amenaza a la sostenibilidad. El análisis se complica cuando se incluyen como elemento fundamental a los bienes esenciales, los inapropiables y los públicos, pues éstos no solamente funcionan en el sistema económico con base en la oferta y la demanda, sino que además forman parte elemental dentro del sistema de supervivencia de los seres vivos.

No obstante lo anterior, existen diversas posturas dentro de la misma economía 
que postulan el crecimiento económico como el medio más eficaz para hacer frente al deterioro ambiental, y quien propone al desarrollo sustentable como la vía adecuada para combinar el bienestar del medio ambiente permitiendo la continuidad del crecimiento y el desarrollo económico.

\section{Debate teórico sobre la relación crecimiento económico y medio ambiente}

Incrementar el producto interno bruto es una de las principales metas de cualquier administración pública nacional; según Samuelson y Nordhaus, "el crecimiento económico continúa siendo un objetivo fundamental de la política económica de los países"; ${ }^{21}$ no obstante el interés y la búsqueda constante de ello, las economías del mundo presentan tasas de crecimiento muy diferentes que las ha llevado a alcanzar un desigual nivel de desarrollo doméstico, quedando en el sistema internacional una clasificación, según el Banco Mundial, de tres tipos de países: desarrollo alto, desarrollo medio y desarrollo bajo.

Field y Field señalan que anteriormente los problemas ambientales se relacionaban con las economías desarrolladas y que se creía "que los países en vías de desarrollo tendrían menos problemas ambientales porque sus tecnologías preindustriales eran más respetuosas con la naturaleza y sus habitantes aún no habían adoptado un estilo de vida materialista". ${ }^{22}$ Sin embargo, los autores afirman que en las últimas décadas las cosas han cambiado, ya que se ha comprobado que los países en desarrollo generan grandes desastres ambientales, lo que implica que los problemas ecológicos para este tipo de economías sean "una cuestión de vida o muerte". Lo anterior lo afirman debido a que los altos niveles de contaminación degradan no sólo el medio ambiente sino los recursos naturales, es decir, su principal insumo productivo, lo que los posiciona como entidades con profundo grado de vulnerabilidad, pues es sabido que en este tipo de economías, especialmente las de desarrollo bajo, el sector primario es el que mayor contribución tiene en el PIB.

Otro problema que según Field y Field se presenta dentro de los países en vías de desarrollo, en el corto y mediano plazos, es que aunque ellos quisieran aspirar a una mejor calidad ambiental no pueden hacerlo porque esto significaría sacrificar el nivel de producción nacional. La afirmación anterior la sostienen mediante una explicación gráfica de las curvas de posibilidades de producción.

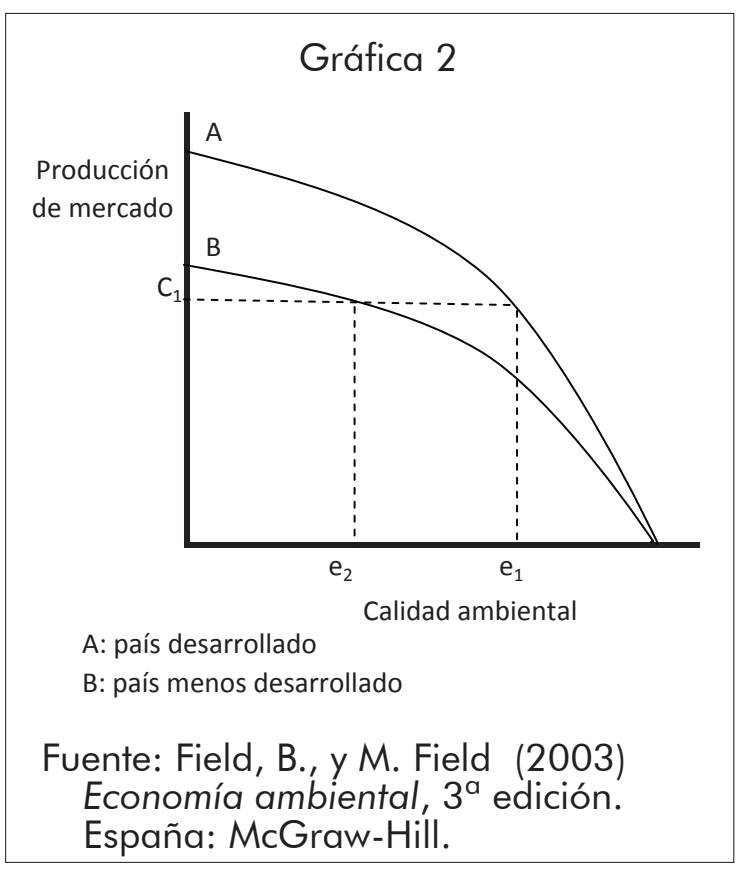

La gráfica 2 presenta, desde una visión estática, la curva de posibilidades de la producción (CPP) de un país desarrollado y otro en vías de desarrollo. La CPPA representa a un país desarrollado y la CPPB corresponde a una economía en desarrollo. "Sea por la 
explotación que se hiciera de los recursos en el pasado, por la presión demográfica o por el uso de tecnología menos avanzada [...] la curva $\mathrm{B}$ se encuentra por debajo de la A" ${ }^{23}$ Si B decidiera alcanzar niveles de producción superiores debe estar dispuesto a sacrificar la calidad de su medio ambiente; en otras palabras, "si quisiera producir una cantidad como $\mathrm{C}_{1}$ tendría que permitir que la calidad se deteriorase hasta el nivel $\mathrm{e}_{2}$. Es decir, el desarrollo económico y la calidad ambiental guardan entre sí una relación de intercambio". ${ }^{24}$ En cambio, el país desarrollado puede permitirse un nivel de producción en $\mathrm{C}_{1} \mathrm{y}$ mantener mayor calidad ambiental $\mathrm{e}_{1}$.

No se debe perder de vista que la gráfica anterior muestra un modelo de naturaleza estática, pues en el largo plazo (el cual se encuentra conectado directamente con el desarrollo económico) se esperarían cambios en el sector productivo como consecuencia de la innovación en la tecnología y en el desprendimiento de la dependencia de los recursos naturales para su economía; así la CPP se desplazaría hacia afuera.

Social y políticamente Grossman afirma "que en la medida que el ingreso se incrementa, la preocupación por el medio ambiente, tanto de gobernadores como de la ciudadanía en general, aumenta y esto induce a una respuesta de política pública que mejore el medio ambiente". ${ }^{25}$ Por su parte Esty señala: "en las naciones acaudaladas, los temas relacionados con la calidad de vida han adquirido una mayor relevancia y la gente siente que puede pagar estándares ambientales más altos". ${ }^{2} 6$

Hasta aquí sólo hemos visto un lado del problema, del cual podríamos concluir que efectivamente los países desarrollados tienen mejor manejo ambiental; sin embargo, vale la pena recordar que los países desarrollados en su proceso de evolución también usaron, de manera indiscriminada, los recursos naturales y al mismo tiempo emitieron grandes cantidades de contaminantes y residuos tóxicos; por lo tanto, se podría interpretar como que en el sistema internacional existe un continuum por el que necesariamente atraviesan los países en el transcurso de su desarrollo. La explicación de esto se encuentra en una propuesta de Samuelson y Nordhaus.

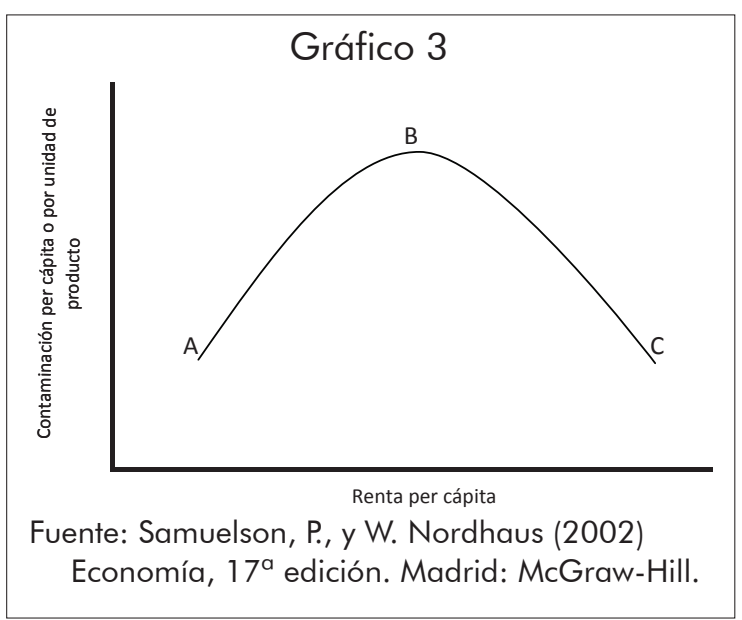

La gráfica 3 muestra el efecto que tiene la contaminación en el crecimiento económico. En los niveles de renta baja del punto A, la agricultura de subsistencia produce poca contaminación. Después, en la fase inicial de desarrollo el crecimiento de la industria pesada sin control de residuos provoca una contaminación per cápita mayor en el punto B. Por último, con la reducción de la contaminación y el desplazamiento de la industria hacia los servicios en los países avanzados, la contaminación disminuye en el punto C. ${ }^{27}$

En la primera mitad de la curva existe una relación positiva entre el deterioro ambiental y el crecimiento económico, es decir el segundo es responsable directo del primero. Empero, según la gráfica a partir de la segunda mitad los niveles de contaminación son decrecientes. Hasta aquí es 
necesario dejar en claro tres cosas: a) en el planeta los países desarrollados son los menos; b) el proceso de desarrollo es bastante complejo, por lo que no existe una receta que se aplique a todos los países y que sea capaz de impulsar el desarrollo, y c) la distribución de la riqueza entre los países $\mathrm{y}$ al interior de ella es muy inequitativa. En este sentido, son pocos los países que alcanzan a desarrollarse. En este punto cabe lanzar una pregunta: ¿soportaría el medio ambiente si todos los países iniciaran su proceso de industrialización? Si no se establece un proyecto diferente del actualmente empleado en el proceso de producción la respuesta sería no, pues el costo sería muy alto.

Por otro lado, la gráfica le resta confiabilidad al hecho de que el país más desarrollado del mundo (Estados Unidos) sea el que emita casi la cuarta parte de los gases de efecto invernadero que se generan en el planeta; y que además a partir de finales de los años sesenta algunos de los países desarrollados trasladaron sus empresas contaminantes (hierro, metales no ferrosos, compuestos químicos industriales, pulpa y papel, etc.) a los países no desarrollados conocidos como paraísos de contaminación. Por lo tanto, parece que en algunos de los países desarrollados no aplica el modelo o son los mismos gobiernos los que manejan el doble discurso (clamar ante sus ciudadanos bienestar ambiental sin adoptar medidas firmes para lograrlo o enviar a otras naciones, con regulaciones ambientales más bajas, las industrias contaminantes).

De acuerdo con la gráfica 3 , la generación de contaminación y el uso de los recursos ambientales es un derecho natural que tienen los países como medio para alcanzar su desarrollo; en este sentido, los países desarrollados no tendrían elementos para debatir o criticar este asunto, pues ellos en el camino hacia su industrialización hicieron lo mismo. Sin embargo, existe el riesgo de que en la defensa de este derecho (del uso de los recursos naturales y de contaminar) el medio ambiente natural colapsara y frenara el crecimiento económico de todo tipo de países, pues recordemos que algunos fenómenos naturales no respetan fronteras, pues son males públicos mundiales; tal es el caso del calentamiento global, de los agujeros en la capa de ozono, la erosión del suelo, las lluvias ácidas, entre otros. De hecho estudios recientes, como el Reporte Stern, mencionan que de no tomarse medidas inmediatas para reducir la emisión de gases de efecto invernadero:

[...] los riesgos y costos en conjunto del cambio climático equivaldría a perder al menos $5 \%$ del producto interno bruto (PIB) cada año, ahora y por siempre. Si un amplio rango de riesgo e impactos son tomados en cuenta, la estimación del peligro podría aumentar a $20 \%$ o más. Por el contrario, el costo de actuar ahora para reducir las emisiones de dióxido de carbono para evitar los peores impactos del cambio climático pueden ser limitados a alrededor del 1\% del PIB por año". ${ }^{28}$

La afirmación anterior es sumamente seria, pues hasta hoy sólo se habían presentado resultados científicos sobre las consecuencias del cambio climático en la naturaleza y su efecto en los seres vivos (inundaciones, erosión del suelo, sequías, escasez de alimentos y agua, tormentas más fuertes, deshielo de los glaciares, entre otros), pero ahora se tienen los primeros estudios sobre el costo económico que traerá este fenómeno. El Reporte Stern anuncia que resulta más económico actuar ahora que ignorar el hecho y actuar después, pues la primera tendría un costo aproximado de $1 \%$ anual, mientras que el no hacer nada traería un costo de entre 5 y $20 \%$ del PIB. 
En suma, resulta casi imposible imaginar lo que pasaría si el medio ambiente limitara el "tan preciado" crecimiento económico, pues además de las desgracias naturales (que generarían desolación, enfermedad y muerte) se sumarían las adversidades sociales tales como altos niveles de desempleo, incremento de las cifras de pobreza, marginación, delincuencia, entre otras. Al respecto, los economistas mantienen una postura optimista al apostar por los avances tecnológicos en la búsqueda de frenar la degradación ambiental y mantener el crecimiento económico.

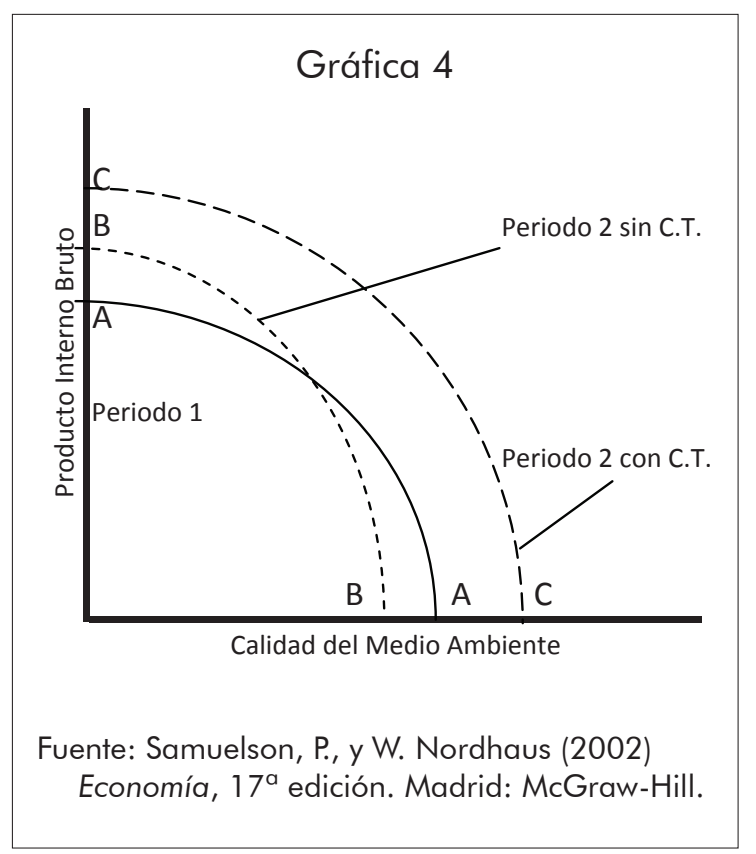

La gráfica 4 señala que las restricciones impuestas por el medio ambiente, es decir la posibilidad de que el medio ambiente limite el crecimiento económico, pueden superarse con nuevas tecnologías. El análisis inicia en el periodo 1 con una frontera de posibilidades de producción (FPP) entre la calidad del medio ambiente y la producción (AA). "El crecimiento económico sin cambio tecnológico (CIT) traslada la FPP a BB. En esta nueva situación la sociedad podría producir más a costa de deteriorar la calidad del medio ambiente". Ésta es la visión pesimista. La situación mejora cuando se incorpora la tecnología (limpia), ${ }^{29}$ la cual desplaza la curva hacia la derecha de la FPP hasta CC; en este punto la sociedad puede consumir más y gozar de un medio ambiente más limpio.

Tal como se presenta en las gráficas 3 y 4, en un futuro, si se innova en tecnología y se aplican políticas ecológicas el sistema productivo no generará contaminación, pero todo esto pareciera mera especulación pues en la actualidad el proceso productivo de algunos países desarrollados, identificados con tecnología de punta, generan altas tasas de contaminación y las medidas políticas y económicas que se aplican no intentan dar una solución real sino que fungen como paliativos; además, cuando hablamos de un futuro sin contaminación como consecuencia de la implementación de la tecnología, ¿en cuánto tiempo se está pensando?, pues desde las últimas décadas los desastres naturales se han estado presentando de manera más constante y con más fuerza. ${ }^{30}$ Es decir, las predicciones emitidas por los especialistas ya están ocurriendo y se pronostica se hagan más severas; en este sentido, ¿cuándo llegará el tan anhelado futuro que mencionan los economistas? Esperemos que no sea demasiado tarde. Indudablemente la situación mundial se ha convertido, tal y como Beck lo mencionó, en una sociedad de riesgo. ${ }^{31}$

Existe la certeza de una crisis ambiental; al mismo tiempo existe la posibilidad de minarla recurriendo a "la eficiencia energética (producir más con menos energía mediante el uso de innovaciones tecnológicas) y al aprovechamiento de tecnología más benigna, como las fuentes de energía renovables". ${ }^{32}$ El problema es que ambas medidas requieren de cuantiosas inver- 
siones económicas que de alguna manera afectarían a algunos sectores de cualquier país; incluso se ha manejado que la protección al medio ambiente genera aumento en los precios de los productos, cierre de fábricas, incremento del desempleo y disminución de la competitividad internacional; entonces, ¿quién correría con los costos? De acuerdo con el Reporte Stern, el poner medidas para frenar el cambio climático requiere de sacrificar el 1\% del PIB anual, pero aunque evidentemente esta medida afectará de manera muy distinta a los países y a pesar de no tener los elementos para realizar un análisis de la manera en que afectaría a cada una de las economías, sí se puede afirmar que si esta medida (del 1\% del PIB) la emplean todos los países, la competitividad a nivel internacional será comparable a la actual.

Sin embargo, si tomamos en cuenta que en la actualidad el sistema económico que rige es, según palabras de Wallerstein, el capitalismo histórico, que se caracteriza por ser un "escenario integrado, concreto, limitado por el tiempo y el espacio, de las actividades productivas dentro del cual la incesante acumulación de capital ha sido el objetivo o ley económica que ha gobernado o prevalecido en la actividad económica fundamental", ${ }^{33}$ entonces resulta poco conveniente para el sistema dejar de acaparar ganancias económicas.

De acuerdo con Wallerstein, para conseguir una constante acumulación de capital el sistema necesita expandirse tanto en materia de producción como en términos geográficos; así:

[...] el capitalismo histórico ha llegado a ser una amenaza para la posibilidad de una existencia futura viable de la humanidad, por haber sido el primer sistema histórico que ha englobado toda la Tierra y que ha expandido la producción y la población más allá de todo lo previamente imaginable". ${ }^{34}$
Definitivamente, para Wallerstein dentro del capitalismo no existe una solución posible para frenar el deterioro ambiental pues esto iría en contra de su propia esencia. Así, el autor afirma que establecer "medidas ecológicas significativas [...] podría ser el golpe de gracia a la viabilidad de la economía-mundo capitalista". ${ }^{35}$

Es decir, el mantener el crecimiento económico apostando en la tecnología limpia y en la aplicación de medidas ecológicas resulta insuficiente en un mundo regido por un sistema económico como el capitalismo, pues éste basa su funcionamiento en el incremento incesante de la producción (lo que implica aumento de consumo) y las ganancias económicas que obtiene de ellas. Así, en esta carrera quien repuntará será siempre la producción.

Desde 1987, como resultado del Informe Brundtland, ${ }^{36}$ se ha venido generando la idea de que la única manera de mantener el desarrollo económico, de gozar de los recursos naturales y del medio ambiente es emplear el desarrollo sustentable, es decir "satisfacer las necesidades del presente sin comprometer la capacidad de las generaciones futuras de satisfacer sus propias necesidades". ${ }^{37}$

De acuerdo con Capalbo, el discurso del desarrollo sustentable "se preocupa en producir en un modo más limpio, se propone consumir recursos renovables, enfatiza en la prevención - si es posible- la minimización de los impactos negativos, pero jamás se menciona la idea de producir menos". ${ }^{38}$ En este sentido no se está cortando el problema de raíz. La propuesta tiene dos inconvenientes: a) de ofrecer el concepto pero no explicar, de manera clara, cómo realizarlo, y b) de mantenerlo bajo el esquema del sistema capitalista, es decir, privilegiando lo económico antes que lo ambiental.

MÉXICO YLACUENCADEL PACÍFICO vol. 10, núm. 30 / septiembre - diciembre de 2007 
Evidentemente tal y como aquí se planteó existen elementos para afirmar la cercana relación entre el crecimiento económico y el medio ambiente, donde el primero en su sistema productivo degrada al segundo. Sin embargo, se carece de los elementos necesarios para afirmar si la ciencia y la tecnología son la panacea que traerán la solución a los problemas ambientales, o si lo que se necesita es un cambio del sistema económico. Por lo pronto en el siguiente apartado se intentará realizar un estudio empírico del caso de Malasia en su proceso de desarrollo económico.

\section{El crecimiento económico y el deterioro ambiental: el caso de Malasia}

Malasia es un país de formación reciente. En 1957 el movimiento político nacionalista de la Federación Malaya alcanzó su independencia de la influencia inglesa. Para esas fechas el sistema económico del país tenía una fuerte dependencia de la explotación de los recursos naturales. El sector primario era el que mayor contribución aportaba a la economía, pues representaba $37 \%$ del PIB, empleaba a 53\% de la fuerza laboral y constituía $89 \%$ de las exportaciones. ${ }^{39}$

En 1969 el gobierno malayo, encabezado por el primer ministro Abdul Razak, elaboró medidas económicas novedosas conocidas como la nueva política económica (NEP), las cuales estaban diseñadas para desarrollar y modernizar al país. La NEP se enfocó en tres objetivos principales: "acelerar el crecimiento, propiciar el avance social de los grupos étnicos más desfavorecidos y modernizar de modo general las estructuras del país. ${ }^{40}$

Desde su etapa de lanzamiento la NEP resultó muy eficaz para la economía del país; el sector productivo dejó de depender mayormente del sector primario para incrementar gradualmente la importancia de sectores como mayor valor agregado como el manufacturero y los servicios. La tasa de crecimiento anual durante el periodo de 1970 a 1976 se mantuvo en un promedio de 8.8\%; en los ochenta se conservó en $4.3 \%$ anual, y durante el periodo de los años noventa y principios del siglo XXI fue de 6.3 por ciento.

\section{Cuadro 1}

Malasia: contribución porcentual de los diferentes sectores en el PIB nacional

\begin{tabular}{cccc}
\hline Años & Agricultura & Industria & Servicios \\
\hline 1960-1980 & 29 & 30 & 41 \\
$1981-1990$ & 20 & 39 & 41 \\
$1991-2000$ & 13 & 42 & 45 \\
\hline
\end{tabular}

Fuente: Banco Mundial (2003) World Development Indicator 2001. Washington, DC: Banco Mundial.

El cuadro 1 muestra la evolución que han tenido los diferentes sectores dentro del PIB nacional. Se puede observar que durante los primeros 20 años del análisis el sector agrícola contribuyó, en promedio, con $29 \%$, la industria con $30 \%$ y los servicios con $41 \%$. Con el correr de los años el peso del sector primario fue minando hasta quedar en $13 \%$, mientras que el de la industria y los servicios fue aumentando. Esto se dio como consecuencia de lo que Pérez y Sierra señalan como parte de la estrategia de la NEP, la cual consideraba que a pesar de que Malasia contaba con muchos productos naturales no iba a vivir de los ingresos producidos por la explotación y exportación de los recursos naturales, sino que se enfocarían hacia procesos más avanzados de valor agregado. ${ }^{41}$

Adicional a la NEP, la aprobación en 1986 de la Ley de Promoción de Inversión 
tuvo un papel fundamental en el proceso de industrialización de Malasia, pues con su implementación se "favorecieron las actividades de investigación y desarrollo, se impulsaron los sectores de hardware y software y se apoyó al sector de los servicios". ${ }^{42}$

Lo anterior constituye una muestra del éxito de la industrialización y la urbanización de Malasia. En este análisis se resalta, especialmente, el hecho de que si bien la naturaleza proveyó a Malasia de preciados recursos naturales (estaño, petróleo, gas, recursos forestales, aceite de palma, cacao, entre otros), los representantes del gobierno rechazaron la cómoda postura de apostar por los recursos abundantes para generar crecimiento económico, sino que los bienes naturales fueron utilizados como medio para la industrialización del país y no como la manera en sí misma de generar crecimiento económico. Cabe reconocer que desde 1970 el gobierno malayo estableció un sistema de monitoreo nacional para medir la calidad del aire y del agua, ${ }^{43}$ lo que nos habla de una preocupación oficial por mantener la salud humana.

Aunque la apuesta de desarrollo no se enfocó en la explotación de los recursos naturales, es evidente que el proceso de industrialización demanda el uso de éstos; prueba de ello es que en la actualidad las reservas de recursos naturales son menores que hace poco más de dos décadas. ${ }^{44}$ En este sentido, aun con las medidas empleadas para proteger la naturaleza, la degradación del ambiente ha sido constante; ejemplo de esto es la disminución del área forestal, ya que en la década de los noventa la superficie era de 116,567 mil hectáreas, para 2005 la cubierta de selva se redujo a sólo 88,495 mil hectáreas, ${ }^{45}$ es decir durante estos 15 años la deforestación fue de alrededor de $0.7 \%$ anual, mientras que la reducción neta fue de más 28 millones de hectáreas.

Otra manera de medir la degradación ambiental es a través de la emisión de gases contaminantes tales como el dióxido de carbono (fluido generado por la quema de combustibles fósiles necesario en el proceso de industrialización). En este sentido, resulta interesante analizar cómo ha sido el desempeño de la emisión de gases en Malasia. Al mismo tiempo, por medio de la observación de la evolución del crecimiento del PIB y del flujo de gases, analizar si existe relación entre éstos, es decir, si el proceso del proceso de desarrollo económico aumenta a niveles similares que la emisión de gases de efecto invernadero, causantes del cambio climático.

En general la década de los noventa y principios de la del 2000 fue muy fructífera para la economía de Malasia, pues este país presentó un crecimiento promedio durante estos 13 años de 6.3\%. Se puede observar en la gráfica 5 que esta economía sufrió una recesión que inició en 1997 y se agudizó en el año siguiente, para volver a retomar la tendencia positiva en 1999. Es importante aclarar que la desaceleración de la economía de Malasia estuvo influida por la llamada crisis asiática, la cual afectó a las economías de mayor crecimiento de la época. Sin embargo, lo importante aquí era verificar que sí se presentó un crecimiento económico. Ahora veamos el comportamiento de los gases contaminantes.

La gráfica 6 muestra la emisión de dióxido de carbono de Malasia durante el periodo 1990-2003. Se puede apreciar que, salvo algunas excepciones, en todos los años se presentó un crecimiento positivo. En los primero cinco años el aumento en la tasa de emisiones fue de $17 \%$. En los dos años posteriores ésta se mantuvo sin cambios representativos, pero a partir de 
Análisis

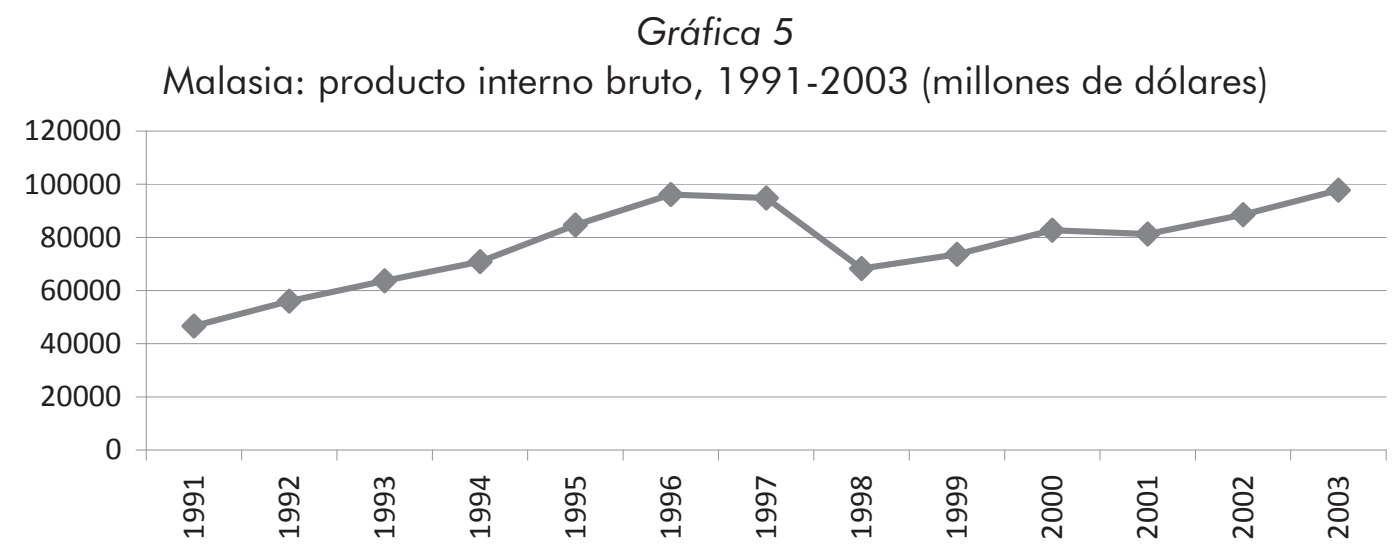

Fuente: elaboración propia con datos del FMI, International Financial Statistics, yearbook 2003-2005.

1998 (coincide con la crisis económica) la producción de gases bajó significativamente (-7\%). En el año 2000 la tendencia retomó su cauce al registrar un crecimiento promedio anual hasta el año 2003 de 10\%. En general, durante este lapso la emisión de gases de efecto invernadero en Malasia aumentó 8 por ciento.

Evidentemente, en el caso de Malasia existe una estrecha relación entre el crecimiento económico y el deterioro ambiental. Incluso el crecimiento económico y la emisión de gases, en términos relativos, crecieron casi a la par (6.3 y 8\% en promedio anual) en este país. Es interesante destacar el punto de la crisis, pues cuando la economía malaya se contrajo también lo hizo la emisión de gases, lo que implica, según los elementos analizados, cercanía entre el proceso de producción y la contaminación del aire.

Grosso modo se presentó el desenvolvimiento de un país con éxito económico reciente, donde el modelo económico em-

\section{Gráfica 6}

Malasia: emisiones de dióxido de carbono, 1990-2003 (mil toneladas)

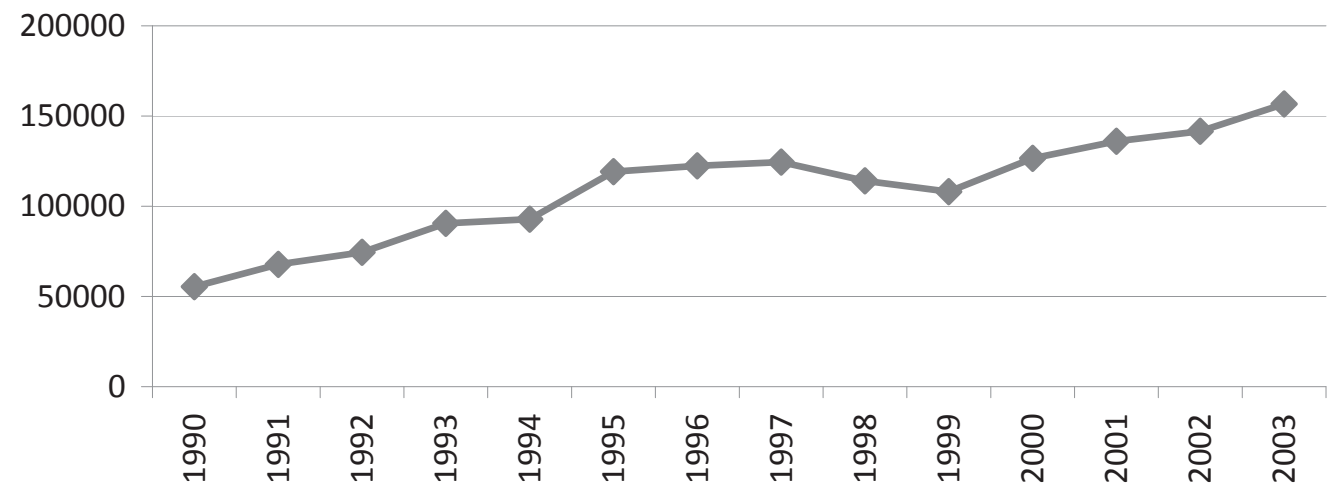

Fuente: elaboración propia con datos de los Indicadores de los objetivos de desarrollo del Milenio, en: http://millenniumindicator.un.org/unsd/mispa/mi_series_resultsd.aspx 
pleado no sólo generó riqueza sino también avances en el campo social de la nación. De Malasia se pueden destacar muchas virtudes, pero en este trabajo sólo se enfocará en tres: a) las medidas económicas para generar crecimiento fueron acertadas, mismas que permearon en cuestiones sociales; b) el no basar su plan de desarrollo en la explotación de los recursos naturales fue un gran acierto, y c) el empezar a emplear medidas de protección ambiental desde los años setenta indica disposición al desarrollo $\mathrm{y}$ bienestar integral.

Se podría pensar que los instrumentos ambientales no tuvieron el mismo triunfo que las medidas económicas (y no se va a refutar la idea), pero cabe señalar que en el proceso de producción la materia prima siempre se obtiene de la naturaleza (aun cuando se empleen bienes intermedios) y que en el transcurso de su transformación éstas generan desperdicios que regresan irremediablemente al medio ambiente. En este sentido, se podría decir que éste es el costo inevitable de la industrialización de un país. Tal y como se presenta el caso de Malasia, el proceso evolutivo de la contaminación aún está en el lado izquierdo de la $U$ invertida de la gráfica 3 .

\section{Conclusiones}

Con base en los elementos analizados se puede establecer que existe una estrecha relación entre el crecimiento económico y el deterioro ambiental, pues el primero depende de los recursos naturales para echar a andar el proceso productivo, mien- tras que el medio ambiente funge como captador de los residuos contaminantes inherentes al sistema de producción. Dos actividades que degradan, de manera constante, la calidad del entorno natural.

Lo peligroso en esto es que no solamente la economía depende de la naturaleza para funcionar, sino que también los seres humanos necesitamos invariablemente de ella para sobrevivir. En este sentido, qué tanto valor tiene que el crecimiento económico ofrezca elevar los estándares de vida de los habitantes en materia de salud, educación, laboral y en el desarrollo personal, si el medio ambiente amenaza con grandes desastres naturales que ponen en riesgo todo lo ofrecido por el crecimiento económico. En esta subasta de ofrecimientos, ¿quién resulta más convincente? ¿A quién le entregaremos el más grande de los tesoros, es decir, la vida misma?

Los economistas son optimistas en cuanto apuestan por la tecnología para hacer frente al detrimento ambiental y es muy posible que se alcancen logros; sin embargo es claro, por un lado, que la tecnología es innovada en los países desarrollados y que el proceso de difusión entre los países requiere de tiempo. Por otro lado, aunque es evidente que la tecnología empleada en el proceso productivo de la actualidad es menos contaminante que en el pasado, aún generan daño al medio ambiente. Asimismo, la tecnología del futuro será mejor que la del presente. Entonces, de lo que se está hablando es de tiempo, mismo que también requiere la naturaleza; en 
este sentido, ¿qué tanto se puede esperar de esta propuesta?

Otro aspecto que no considera el modelo economicista son los costos económicos de la innovación y el empleo de la tecnología limpia. ¿Quién los asumirá?, pues recordemos que el sistema económico capitalista (que rige en la actualidad) se basa en el crecimiento constante de la producción y el consumo, mismos que lo llevan a la obtención de ganancias económicas. Así, ¿cómo se podrá combinar la disminución de las ganancias monetarias (producto del empleo de medidas ecológicas) y el mantenimiento de la lógica capitalista? Según palabras de Wallerstein, no hay salida favorable para el restablecimiento de la salud medioambiental dentro del sistema histórico existente.

Sin embargo, según se apreció en el trabajo, existen medidas propuestas dentro del Reporte Stern para hacerle frente al cambio climático (no es el único problema ambiental pero sí uno de los más apremiantes por la magnitud de su alcance), el cual propone asumir un costo equivalente a $1 \%$ del PIB para emplear medidas eficientes que logren reducir los niveles de gases de efecto invernadero causantes de cambio climático, y con ello los efectos adversos a la naturaleza y a la sociedad. De lo contrario, señala el Reporte, el no hacer nada (que es lo que en general ha venido ocurriendo) implicaría, a mediano y largo plazos, un costo de entre 5 y $20 \%$ del PIB. Evidentemente para cualquier gobierno la primera opción es la más viable, pues se considera que el sistema económico puede seguir funcionando con esto.

El no tomar medidas ahora que requieren el mínimo de gastos, las circunstancias les obligarían, en un futuro no muy lejano, a realizarlo sin mayor opción (cuando los costos serán mayores), pues ése sería el único camino para hacer frente a los desastres naturales, junto con sus consecuencias. Llegados a este punto, estarían las condiciones dadas para que sin más el sistema capitalista colapse.

El análisis particular de Malasia es la muestra real de que el camino hacia la industrialización viene acompañado de deterioro ambiental. Desde finales de los años sesenta el crecimiento económico de este país ha sido constante, y su dificultad para mantener el bienestar del medio ambiente también. mivg

\section{Notas}

1. UNEP (2003) Global Environment Outlook, disponible en: http://www.unep.org/geo/geo3/ spanish/086.htm

The World Bank (2006) World Development Indicators. Washington: The World Bank.

2. Field, y Field, 2003: 3-16.

3. Ídem.

4. Wallerstein, 1998.

5. Samuelson y Nordhaus, 2002: 491.

6. "The Economics of Climate Change", Stern Review.

7. Vincent y Mohamed, 1997: 1 y 2.

8. Samuelson y Nordhaus, 2002: 319.

9. "Son las situaciones en las que la producción o el consumo impone a otros costes o beneficios que no son compensados" (ibíd.: 320-324).

10. Ludevid, 1999: 97.

11. Los recursos renovables se clasifican en dos grupos: los recursos biológicos y los recursos fluidos. Los primeros consisten en la las especies de plantas y animales. Ellos pueden ser capaces de autorrenovación, sin embargo si su explotación va más allá de su capacidad de regeneración éstos tienden a la desaparición. Mientras que recursos fluidos son expedidos por la atmósfera y la circulación hidráulica (Hussen, 2004: xxvi).

12. Éstos se clasifican en dos: los reciclables y los no reciclables (ídem).

13. Samuelson y Nordhaus, 2002: 320 .

14. Kaul, Grunberg y Stern, 2000: 4.

15. Samuelson y Nordhaus, 2002: 324 y 325.

16. Ídem.

17. Field y Field, 2003: 27.

18. Ibíd.: 28.

19. Ídem. 
20. Cuando su explotación es más rápida que su capacidad de renovación.

21. Samuelson y Nordhaus, 2002: 491.

22. Field y Field, 2003: 451.

23. Ibíd.: 454.

24. Ídem.

25. Grossman, citado en Banco Mundial, 2002: 114.

26. Esty, 2001: 28.

27. Samuelson y Nordhaus, 2002: 319.

28. Stern, N. (2006) Stern Review on the economics of climate change. En http://www.hm-treasury. gov.uk/independent_reviews/stern_review_economics_climate_change/stern_review_report. $\mathrm{cfm}$

29. "La introducción de equipo para extraer y quemar carbón bajo en azufre, la obligación de llevar dispositivos en los automóviles, controlar la contaminación o el desarrollo de energía solar, etcétera" (Samuelson y Nordhaus, 2002: 497).

30. Gore, 2007.

31. Beck, U., citado en Lezama, J. (2001) El medio ambiente hoy, temas cruciales del debate contemporáneo. México: El Colegio de México.

32. Uranga, 2006: 178 y 179.

33. Wallerstein, 1988: 7.

34. Wallerstein, 1998. http://www.inisoc.org/ecologia.htm

35. Ídem.

36. Cuyos objetivos principales son: examinar los temas críticos de desarrollo y medio ambiente; formular propuestas realistas al respecto; proponer nuevas formas de cooperación internacional.

37. Barcena, Ibarra y Zubiaga, 2000: 12.

38. Capalbo, 2000: 16.

39. Vincent y Mohamed, 1997: 10.

40. Pérez y Sierra, 2004: 134.

41. Ibíd.: 135 .

42. Ídem.

43. Vincent y Mohamed, 1997: 2.

44. (Vincent J. \& Mohamed R., 1997: 30)

45. Tablas mundiales de la Evaluación de los recursos forestales mundiales, en: http://www. fao.org/forestry/site/fra2005/es/

\section{Bibliografía}

Bárcena, I., P. Ibarra, y M. Zubiaga (2000) Desarrollo sostenible: Un concepto polémico. País Vasco: Editorial de la Universidad del País Vasco.

Banco Mundial (2002) Globalización, crecimiento y pobreza. Colombia: Alfaomega.

Capalbo, L. (2000) "Desarrollo: del dominio material al dominio de las ilimitadas potencialidades humanas", en M. Acevedo, A. Elizalde, et al., El resignificado del desarrollo. Buenos Aires: UNIDA.

Coxhead, I., y S. Jayasuriya (2003) The Open Economy and The Environment: Development, Trade and Resources in Asia. Massachusetts: Edward Elgar Publishing.

Esty, C. (2001) El reto ambiental de la Organización Mundial del Comercio. Barcelona: Gedisa.

FAO (2005) Tablas mundiales de la evaluación de los recursos forestales mundiales. Disponible en: http:// www.fao.org/forestry/site/fra2005/es/

Field, B., y M. Field (2003) Economía ambiental, $3^{a}$ edición. España: McGraw-Hill.

Gore, A. (2007) Una verdad incómoda. Barcelona: Gedisa.

Hussen, A. (2004) Principles of Environmental Economics. Nueva York: Routledge.

Fondo Monetario Internacional (FMI) (2005) International Financial Statistics. Washington: FMI.

Kaul, I., I. Grunberg, y M. Stern (2000) "Definición de bienes públicos mundiales", en I. Kaul, I. Grunberg, y M. Stern (eds.), Bienes públicos mundiales: la cooperación internacional en el siglo XXI. México: Oxford.

Lezama, J. (2001) El medio ambiente hoy, temas cruciales del debate contemporáneo. México: El Colegio de México.

Pérez, A., e I. Sierra (2004) "Relaciones étnicas y desarrollo económico en Malasia", Política y Cultura, núm. 21. México: UNAM. Disponible en: http:// scielo.unam.mx/pdf/polcul/n21/n21a09.pdf

Samuelson, P., y W. Nordhaus (2002) Economía, 17ª edición. Madrid: McGraw-Hill.

Stern, N. (2006) Stern Review: The Economic of Climate Change. Disponible en: http://www.hm- treasury. gov.uk/independent_reviews/stern_review_economics_climate_change/stern_review_report.cfm

Banco Mundial (2006) World Development Indicators. Washington: Banco Mundial.

Vincent, J., y R. Mohamed (1997) Environment and Development in a Resource-Rich Economy, Malaysia Under the New Economic Policy. Estados Unidos: Harvard University Press.

UNEP (2003) Global Environment Outlook. Disponible en: http://www.unep.org/geo/geo3/spanish/086. htm

Uranga, A. (2006) "Medio ambiente y energía renovable: una perspectiva compleja", en G. Arroyo (coord.), La dinámica mundial del siglo XXI, revoluciones, procesos, agentes y transformaciones. México: Cenzontle.

Wallerstein, I. (1998) "Ecología y costes de producción capitalistas: no hay salida", Iniciativa Socialista, núm. 50. California: Universidad de California. Disponible en: http://www.inisoc.org/ecologia. htm

Wallerstein, I. (1988) El capitalismo Histórico. México: Siglo XXI Editores.

MÉXICO YLACUENCADEL PACÍFICO vol. 10, núm. 30 / septiembre-diciembre de 2007 